\title{
Counting and Discrete Morse Theory
}

\section{Andrew Sack \\ University of Florida}

Faculty mentor: Kevin Knudson, Department of Mathematics

\begin{abstract}
We examine two classes of enumerating discrete Morse functions on graphs. The first, gradient vector fields, is examined heavily in the literature. The second examines discrete Morse functions with codomain in $\{1, \ldots, \mathrm{k}\}$. We give formulae for the number of discrete Morse functions on specific classes of graphs (line, cycle, and bouquet of circles).

Keywords: discrete Morse theory
\end{abstract}

\section{Introduction}

Discrete Morse theory was created by Robin Forman in 1995 as discretization of Morse Theory. (Forman, 1998) Discrete Morse theory deals with so-called discrete Morse functions on regular CW-complexes, analogous to Morse functions on smooth manifolds. Under Forman's definitions, there are many analogous theorems in discrete Morse theory from smooth Morse theory. Discrete Morse theory has found applications in a broad range of topics including topological denoising (Bauer, Lange, C. \& Wardetzky, 2012) and mesh compression (Lewiner, et al., 2004).

In this paper we only deal with 1-dimensional regular $\mathrm{CW}$-complexes, i.e. loop-free undirected (multi-)graphs. Any time the word "graph" is used in this paper it will be of this type.

Definition 1.1. Let $G$ be a graph. A discrete Morse function $f: G \rightarrow R$ is a function that assigns values to the edges and the vertices of $G$ such that the following two conditions are met:

(1) For any vertex $v \in G$, the number of incident edges e such that $f(v) \geq f(e)$ is at most one.

(2) For any edge $e \in G$, the number of incident vertices $v$ such that $f(v) \geq f(e)$ is at most one.

For any non-trivial graph, there are infinitely many discrete Morse functions given by assigning any constant value $m$ to every vertex and any other constant $n>m$ to every edge. To 
meaningfully count the number of discrete Morse functions on a graph, we must introduce an equivalence relation on discrete Morse functions or otherwise restrict the set of discrete Morse functions. One equivalence relation on discrete Morse functions that has been studied extensively is the gradient vector field.(Bauer, et al.)(Chari \& Joswig, 2005)(Forman, 1998)(Lewiner, et al., 2004)

In Corollaries 2.3.1 and 2.3.2 we give formulas for the number of gradient vector fields on $\mathrm{P}_{\mathrm{n}}$, the path graph on $n$ vertices and for $\mathrm{C}_{\mathrm{n}}$, the cycle graph on $n$ vertices. We also give an algorithm to compute the number of discrete Morse functions with codomain in $\{1, \ldots, n\}$ on both $\mathrm{P}_{\mathrm{n}}$ and $\mathrm{C}_{\mathrm{n}}$.

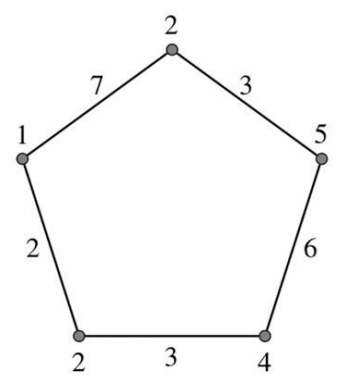

Figure 1. A discrete Morse function on $C_{5}$

\section{Gradient Vector Fields}

Definition 2.1. A discrete vector field is a set of pairs of incident vertices and edges such that no vertex or edge appears in more than one pair.

A common visualization for a discrete vector field is to draw an arrow from the vertex to the edge for any pair in the vector field.

Definition 2.2. Let $V$ be a discrete vector field. $A V$-path is a sequence of vertices and edges $v_{1}, e_{1}, v_{2}, e_{2}, \ldots, e_{n-1}, v_{n}$ such that $v_{i}$ and $v_{i+1}$ are both incident to $e_{i}$ and $\left(v_{i}, e_{i}\right) \in V$.

Definition 2.3. Let $V$ be a vector field on a graph $G$. A vertex or edge is said to be critical if it is not in any pair in $V$.

Definition 2.4. $A V$-path is called closed if $v_{l}=v_{n}$.

Definition 2.5. Let $G$ be a graph and let f be a discrete Morse function on G. The gradient vector field off is the set $\{\{v, e\} \mid v$ and $e$ are incident and $f(v) \geq f(e)\}$.

Gradient vector fields are discrete vector fields.(Forman, 1998) It is known that a vector field $V$ is a gradient vector field if and only if $V$ contains no closed $V$-paths of length greater than 1 . That 
is, if $V$ contains no closed $V$-paths of length greater than 1, then there exists a discrete Morse function $f$ whose gradient vector field is equal to $V$. In Proposition 3.3 of (Chari \& Joswig, 2005), the authors note that the number of discrete gradients on a graph is equal to the number of rooted forests of the graph.

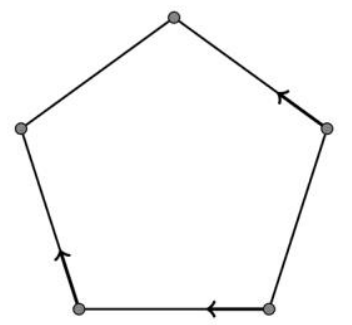

Figure 2. The gradient vector field of Figure 1

Definition 2.6. Let $G$ be a graph. For vertices $u, v \in G$ we define the following six functions:

- $\quad \mu(u)=$ the number of discrete vector fields on $G$ such that $u$ is critical.

- $\quad v(u)=$ the number of discrete vector fields on $G$ such that $u$ is not critical.

- $\mu_{\mu}(u, v)=$ the number of discrete vector fields on $G$ such that both $u$ and $v$ are critical. SİT

- $\mu_{v}(u, v)=$ the number of discrete vector fields on $G$ such that $u$ is critical but $v$ is not

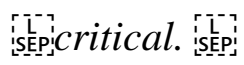

- $\quad v_{v}(u, v)=$ the number of discrete vector fields on $G$ such that neither $u$ nor $v$ is critical. SİT

- $v_{\mu}(u, v)=\mu_{v}(v, u)=$ the number of discrete vector fields on $G$ such that $u$ is not critical

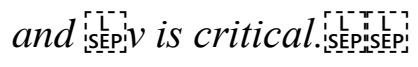

Note that $\mu(u, v)=\mu_{\mu}(u, v)+\mu_{v}(u, v)$ and $v(u, v)=v_{\mu}(u, v)+v_{v}(u, v)$. 
Definition 2.7. Let $(G, u)$ and $(H, v)$ be graphs with distinguished points. Define $(G, u) \vee(H, v)$ to be the graph with $u$ and $v$ identified, i.e. the wedge sum of the graphs. For convenience, we will refer to this as $G \vee H$.

Proposition 2.8. Let $(G, u)$ and $(H, v)$ be graphs with vertices $a \in G$ and $b \in H$. Let $a^{\prime}, b^{\prime}$ and $u^{\prime}$ be the images of $a, b$, and $u$ in $G \vee H$ respectively. Then the following formulae hold:

- $\mu_{\mu}\left(a^{\prime}, b^{\prime}\right)=\mu_{\mu}(a, u) \mu_{\mu}(v, b)+\mu_{\mu}(a, u) v_{\mu}(v, b)+\mu_{v}(a, u) \mu_{\mu}(v, b)$

- $\mu_{v}\left(a^{\prime}, b^{\prime}\right)=\mu_{\mu}(a, u) \mu_{v}(v, b)+\mu_{\mu}(a, u) v_{v}(v, b)+\mu_{v}(a, u) \mu_{v}(v, b)$

- $v_{\mu}\left(a^{\prime}, b^{\prime}\right)=v_{\mu}(a, u) \mu_{\mu}(v, b)+v_{\mu}(a, u) v_{\mu}(v, b)+v_{v}(a, u) \mu_{\mu}(v, b)$

- $v_{v}\left(a^{\prime}, b^{\prime}\right)=v_{\mu}(a, u) \mu_{v}(v, b)+v_{\mu}(a, u) v_{v}(v, b)+v_{v}(a, u) \mu_{v}(v, b)$

- $\mu\left(u^{\prime}\right)=\mu(u) \mu(v)$

- $\quad v\left(u^{\prime}\right)=\mu(u) v(v)+v(u) \mu(v)$

Note: This proposition holds if we replace the words "discrete vector field" by "gradient vector field" in Definition 2.6

Proof. This follows immediately from the observation that any vector field on $G \vee H$ induces a vector field on $G$ and on $H$. Vector fields on $G$ and $H$ induce vector fields on $G \vee H$ only if at least one of $u$ or $v$ is critical. The last two formulae are sums of the previous formulae. The note at the end is true because the wedge operation cannot form new cycles.

Proposition 2.9. Let $G$ be a graph with fixed distinct vertices $u, v \in G$. Let $G^{\prime}$ be the graph with an additional edge between $u$ and $v$ and let $u^{\prime}$ and $v^{\prime}$ be the images of $u$ and $v$ in $G^{\prime}$ respectively. Then:

- $\mu_{\mu}\left(u^{\prime}, v^{\prime}\right)=\mu_{\mu}(u, v)$

- $\mu_{\mu}\left(u^{\prime}, v^{\prime}\right)=\mu_{\mu}(u, v)$

- $\mu_{v}\left(u^{\prime}, v^{\prime}\right)=\mu_{\mu}(u, v)+\mu_{v}(u, v)$

- $\quad v_{v}\left(u^{\prime}, v^{\prime}\right)=v_{v}(u, v)+\mu_{v}(u, v)+v_{\mu}(u, v)$

- $v_{\mu}\left(u^{\prime}, v^{\prime}\right)=\mu_{\mu}(u, v)+v_{\mu}(u, v)$ 
Proof. We note that in a $K_{2}$ (that is, two vertices connected by an edge), there are three vector fields:

(1) Both vertices are critical.

(2) Vertex $u$ is critical and vertex $v$ is not critical.

(3) Vertex $v$ is critical and vertex $u$ is not critical.

(4) Let $V$ be the set of all vector fields on $G$ and let $V^{\prime}$ be the set of all vector fields on $G^{\prime}$. We may apply the following operations to elements of $V$ :

(1) If $u$ and $v$ are both critical, the new edge may either be critical, be paired with $u$ or be paired with $v$. In the first case, this adds to $\mu_{\mu}\left(u^{\prime}, v^{\prime}\right)$, in the second case this adds to $v_{\mu}\left(u^{\prime}, v^{\prime}\right)$ and in the third case this adds to $\mu_{v}\left(u^{\prime}, v^{\prime}\right)$.

(2) If $u$ is critical but not $v$, the new edge may either be critical or be paired with $u$. In the first case this adds to $\mu_{v}\left(u^{\prime}, v^{\prime}\right)$ and in the second case this adds to $v_{v}\left(u^{\prime}, v^{\prime}\right)$. iscepi]

(3) If $v$ is critical but not $u$, the new edge may either be critical or be paired with $v$. In the first case this adds to $v_{\mu}\left(u^{\prime}, v^{\prime}\right)$ and in the second case this adds to $v_{v}\left(u^{\prime}, v^{\prime}\right)$, sép.]

(4) If neither $u$ nor $v$ is critical, the new edge must be critical and this adds to $v_{v}\left(u^{\prime}, v^{\prime}\right)$. ĩ'

Case (1) occurs $\mu_{\mu}(u, v)$ times, case (2) occurs $\mu_{v}(u, v)$ times, case (3) occurs $v_{\mu}(u, v)$ times, and case (4) occurs $v_{v}(u, v)$ times. Each case is disjoint, and no two cases overlap. Furthermore, these give all of $V^{\prime}$. Hence the formulae above can be attained by adding up the cases.

Proposition 2.10. Let $P_{n}$ be the path graph on $n$ vertices and let $u_{n}$ be the first vertex and $v_{n}$ be the last vertex. Then the following formulae hold:

- $\mu_{\mu}\left(u_{n}, v_{n}\right)=F_{2 n-2}$

- $\mu_{v}\left(u_{n}, v_{n}\right)=v_{\mu}\left(u_{n}, v_{n}\right)=F_{2 n-3}$

- $v_{v}\left(u_{n}, v_{n}\right)=F_{2 n-4}$

where $F_{i}$ is the $i$-th Fibonacci number. 
Proof. Consider $P_{n}$ and attach a $P_{2}$ with vertices $x$ and $y$ as in Proposition 2.8 by vertex $x$.

Define $\vec{w}_{n+1}=\left(\begin{array}{c}\mu_{\mu}\left(u_{n+1}, v_{n+1}\right) \\ \mu_{v}\left(u_{n+1}, v_{n+1}\right) \\ v_{\mu}\left(u_{n+1}, v_{n+1}\right) \\ v_{v}\left(u_{n+1}, v_{n+1}\right)\end{array}\right)$

Then

$\vec{w}_{n+1}=\left(\begin{array}{c}\mu_{\mu}\left(u_{n}^{\prime}, y^{\prime}\right) \\ \mu_{v}\left(u_{n}^{\prime}, y^{\prime}\right) \\ v_{\mu}\left(u_{n}^{\prime}, y^{\prime}\right) \\ v_{v}\left(u_{n}^{\prime}, y^{\prime}\right)\end{array}\right)$

$=\left(\begin{array}{cccc}\mu_{\mu}(x, y)+v_{\mu}(x, y) & \mu_{\mu}(x, y) & 0 & 0 \\ \mu_{v}(x, y)+v_{v}(x, y) & \mu_{\mu}(x, y) & 0 & 0 \\ 0 & 0 & v_{\mu}(x, y)+\mu_{\mu}(x, y) & \mu_{\mu}(x, y) \\ 0 & 0 & \mu_{v}(x, y)+v_{v}(x, y) & \mu_{v}(x, y)\end{array}\right)\left(\begin{array}{c}\mu_{\mu}\left(u_{n}, v_{n}\right) \\ \mu_{v}\left(u_{n}, v_{n}\right) \\ v_{\mu}\left(u_{n}, v_{n}\right) \\ v_{v}\left(u_{n}, v_{n}\right)\end{array}\right)$

$=\left(\begin{array}{llll}2 & 1 & 0 & 0 \\ 1 & 1 & 0 & 0 \\ 0 & 0 & 2 & 1 \\ 0 & 0 & 1 & 1\end{array}\right)\left(\begin{array}{l}\mu_{\mu}\left(u_{n}, v_{n}\right) \\ \mu_{v}\left(u_{n}, v_{n}\right) \\ v_{\mu}\left(u_{n}, v_{n}\right) \\ v_{v}\left(u_{n}, v_{n}\right)\end{array}\right)$

Let $K=\left(\begin{array}{llll}2 & 1 & 0 & 0 \\ 1 & 1 & 0 & 0 \\ 0 & 0 & 2 & 1 \\ 0 & 0 & 1 & 1\end{array}\right)$

Hence $\overrightarrow{\mathrm{w}}_{n}=K^{(n-2)} \overrightarrow{\mathrm{w}}_{2}=K^{(n-2)}\left(\begin{array}{l}1 \\ 1 \\ 1 \\ 0\end{array}\right)$

Finally, note that $K^{3}=3 K^{2}-K$, and hence $\vec{w}_{n}=3 \vec{w}_{n-1}-\vec{w}_{n-2}$. The above formulae satisfy this recurrence relation and have the appropriate initial conditions.

Corollary 2.10.1. The number of discrete vector fields on $P_{n}$ is $F_{2 n}$. 
Proof. $F_{2 n-2}+F_{2 n-3}+F_{2 n-3}+F_{2 n-4}=F_{2 n-1}+F_{2 n-2}=F_{2 n}$

Corollary 2.10.2. The number of discrete vector fields on $C_{n}$ is $L_{2 n}$, where $L_{k}$ is the $k$-th Lucas number. The number of gradient vector fields on $C_{n}$ is $L_{2 n}-2$.

Proof. Apply Proposition 2.9 to $P_{n}$ and attach an edge to connect the endpoints $u$ and $v$. Then the number of discrete vector fields on $C_{n}$ is

$$
\begin{aligned}
\mu\left(u^{\prime}\right)+v\left(u^{\prime}\right) & =\left[\mu_{\mu}\left(u^{\prime}, v^{\prime}\right)+\mu_{v}\left(u^{\prime}, v^{\prime}\right)\right]+\left[v_{\mu}\left(u^{\prime}, v^{\prime}\right)+v_{v}\left(u^{\prime}, v^{\prime}\right)\right] \\
& =\left[\mu_{\mu}(u, v)+\mu_{\mu}(u, v)+\mu_{v}(u, v)\right]+\left[v_{v}(u, v)+\mu_{v}(u, v)+v_{\mu}(u, v)+\mu_{\mu}(u, v)\right. \\
& \left.+v_{\mu}(u, v)\right] \\
& =\left[F_{2 n-2}+F_{2 n-2}+F_{2 n-3}\right]+\left[F_{2 n-4}+F_{2 n-3}+F_{2 n-3}+F_{2 n-2}+F_{2 n-3}\right] \\
& =\left[F_{2 n}\right]+\left[F_{2 n-2}+F_{2 n-1}+F_{2 n-3}\right]=\left[F_{2 n}\right]+\left[2 F_{2 n-1}\right]=F_{2 n+1}+F_{2 n-1}=L_{2 n}
\end{aligned}
$$

Exactly two of these discrete vector fields contain closed $V$ - paths, corresponding to the two paths that go around the circle. Hence the number of gradient vector fields is $L_{2 n}-2$.

Comparing this with the formula given in (Chari \& Joswig, 2005) we find that

$$
L_{2 n}=\sum_{i=0}^{n} \frac{2 n}{2 n-i}\left(\begin{array}{c}
2 n-i \\
i
\end{array}\right)
$$

The number of gradient vector fields on $C_{n}$ for $n \in\{2, \ldots, 6\}$ is $5,16,45,121,320$.

Corollary 2.10.3. The number of gradient vector fields on a bouquet of $n$ circles where the $k$-th circle has $a_{k}$ vertices is

$$
\prod_{i=1}^{n}\left(F_{2 i}-2\right)+\sum_{i=1}^{n}\left(2 F_{2 i-1}\right) \prod_{j \in[1 . . n] \backslash\{i\}}\left(F_{2 j}-2\right)
$$

Proof. Apply Proposition 2.8.

\section{Discrete Morse $\boldsymbol{k}$-Functions}


Definition 3.1. A discrete Morse $k$-function is a discrete Morse function with codomain in $\{1,2$, $\ldots, k\}$.

Discrete Morse $k$-functions can be thought of as placing a digital sensor on each cell that can only take $k$ different possible values. For non-trivial graphs, there is only one discrete Morse 2function. We aim to count the number of discrete Morse $k$-functions on a graph. For several classes of graph we make the following definitions:

Definition 3.2. Let $G$ be a graph and $k \in \mathrm{Z}^{+}$fixed. For vertices $u, v \in G$ we define the following four $k \times k$ matrices:

- $M_{M}(u, v)=$ the matrix such that entry $i, j$ is the number of discrete Morse $k$-functions $f$ such that $f(u)=i, f(v)=j$ and both $u$ and $v$ are critical.

- $\quad M_{N}(u, v)=$ the matrix such that entry $i, j$ is the number of discrete Morse $k$-functions $f$ such that $f(u)=i, f(v)=j$, $u$ is critical and $v$ is not critical.

- $\quad N_{N}(u, v)=$ the matrix such that entry $i, j$ is the number of discrete Morse $k$-functions $f$ such that $f(u)=i, f(v)=j$ and neither $u$ nor $v$ is critical. istep?

- $\quad N_{M}(u, v)=$ the matrix such that entry $i, j$ is the number of discrete Morse $k$-functions $f$ such that $f(u)=i, f(v)=j$, $u$ is not critical and $v$ is critical.

Proposition 3.3. Let $(G, u)$ and $(H, v)$ be graphs with distinsuihed vertices and let $a \in G$ and $b \in$ $H$. Let $a^{\prime}, b^{\prime}$ be the images of $a, b$ in $G \vee H$ respectively. Then the following formulae hold:

- $\quad M_{M}\left(a^{\prime}, b^{\prime}\right)=M_{M}(a, u) M_{M}(v, b)+M_{M}(a, u) N_{M}(v, b)+M_{N}(a, u) M_{M}(v, b)$

- $\quad M_{N}\left(a^{\prime}, b^{\prime}\right)=M_{M}(a, u) M_{N}(v, b)+M_{M}(a, u) N_{N}(v, b)+M_{N}(a, u) M_{N}(v, b)$ SEEP

- $\quad N_{M}\left(a^{\prime}, b^{\prime}\right)=N_{M}(a, u) M_{M}(v, b)+N_{M}(a, u) N_{M}(v, b)+N_{N}(a, u) M_{M}(v, b)$

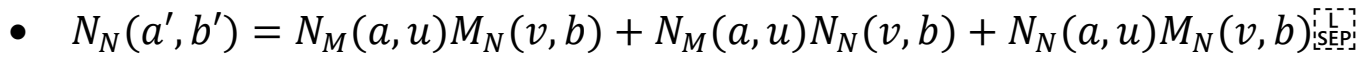

Proof. A discrete Morse function on $G$ and a discrete Morse function on $H$ form a discrete Morse function in the quotient only if the vertices agree in value and no more than one edge takes a value greater than a vertex. is[ip? 
Proposition 3.4. Let $a$ and $b$ be the vertices of $P_{2}$. The following formulae hold:

- The $i, j$ entry in $M_{M}(a, b)$ is $k-\max (i, j)$. [is

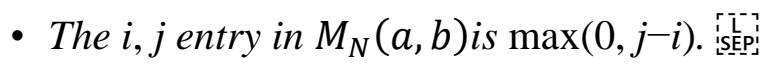

- The $i, j$ entry in $N_{M}(a, b)$ is $\max (0, i-j)$. is ispep

- The $i, j$ entry in $N_{N}(a, b)$ is 0 . istep:

\section{Proof.}

- The $i, j$ entry in $M_{M}(a, b)$ is the number of integers in $\{1, \ldots, k\}$ greater than both $i$ and $j$. sept

- The $i, j$ entry in $M_{N}(a, b)$ is the number of integers in $\{1, \ldots, k\}$ greater than $i$ and less than or equal to $j$. isspep?

- The $i, j$ entry in $N_{M}(a, b)$ is the number of integers in $\{1, \ldots, k\}$ less than or equal to $i$ and iscepigreater than $j$. [is

- The $i, j$ entry in $N_{N}(a, b)$ is 0 because the value of the edge cannot be less than or equal to

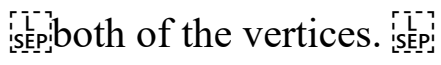

Proposition 3.4. The following is an algorithm with proof to count the number of discrete Morse $k$-functions on $P_{n}$ and on $K_{n}$.

Let $a_{n}$ and $b_{n}$ the initial and terminal vertices of $P_{n}$ for $n \geq 2$. By Proposition 3.3, the following formulae hold:

$$
\begin{aligned}
& M_{M}\left(a_{n+1}, b_{n+1}\right)=M_{M}\left(a_{2}, b_{2}\right) M_{M}\left(a_{n}, b_{n}\right)+M_{M}\left(a_{2}, b_{2}\right) N_{M}\left(a_{n}, b_{n}\right)+M_{N}\left(a_{2}, b_{2}\right) M_{M}\left(a_{n}, b_{n}\right) \\
& M_{N}\left(a_{n+1}, b_{n+1}\right)=M_{M}\left(a_{2}, b_{2}\right) M_{N}\left(a_{n}, b_{n}\right)+M_{M}\left(a_{2}, b_{2}\right) N_{N}\left(a_{n}, b_{n}\right)+M_{N}\left(a_{2}, b_{2}\right) M_{N}\left(a_{n}, b_{n}\right) \\
& N_{M}\left(a_{n+1}, b_{n+1}\right)=N_{M}\left(a_{2}, b_{2}\right) M_{M}\left(a_{n}, b_{n}\right)+N_{M}\left(a_{2}, b_{2}\right) N_{M}\left(a_{n}, b_{n}\right)+N_{N}\left(a_{2}, b_{2}\right) M_{M}\left(a_{n}, b_{n}\right) \\
& N_{N}\left(a_{n+1}, b_{n+1}\right)=N_{M}\left(a_{2}, b_{2}\right) M_{N}\left(a_{n}, b_{n}\right)+N_{M}\left(a_{2}, b_{2}\right) N_{N}\left(a_{n}, b_{n}\right)+N_{N}\left(a_{2}, b_{2}\right) M_{N}\left(a_{n}, b_{n}\right)
\end{aligned}
$$




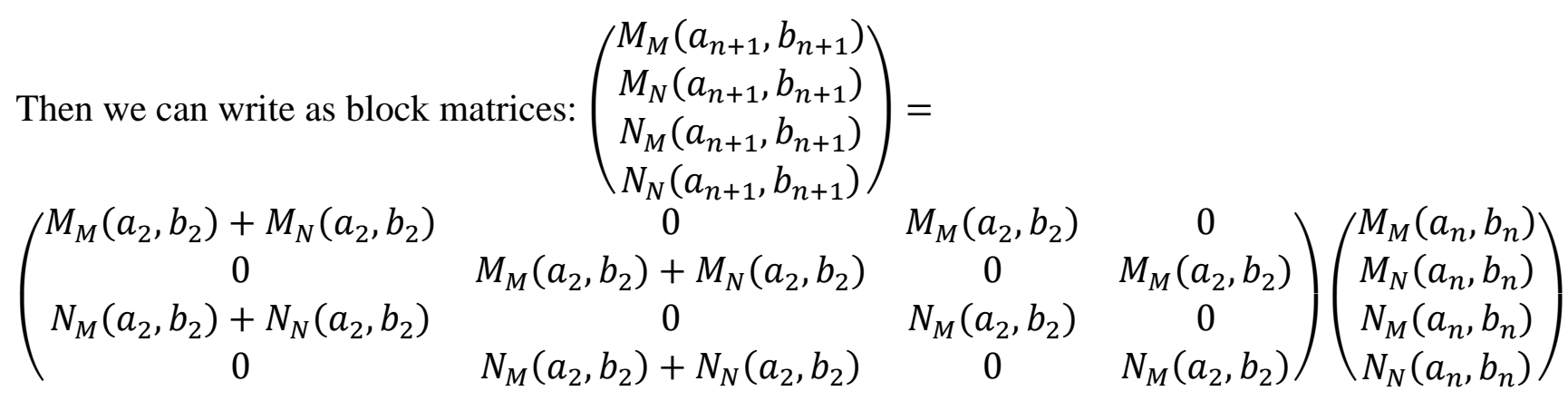

We can simplify with $N_{N}\left(a_{2}, b_{2}\right)=0$ to get

$$
\left(\begin{array}{cccc}
M_{M}\left(a_{2}, b_{2}\right)+M_{N}\left(a_{2}, b_{2}\right) & 0 & M_{M}\left(a_{2}, b_{2}\right) & 0 \\
0 & M_{M}\left(a_{2}, b_{2}\right)+M_{N}\left(a_{2}, b_{2}\right) & 0 & M_{M}\left(a_{2}, b_{2}\right) \\
N_{M}\left(a_{2}, b_{2}\right) & 0 & N_{M}\left(a_{2}, b_{2}\right) & 0 \\
0 & N_{M}\left(a_{2}, b_{2}\right) & 0 & N_{M}\left(a_{2}, b_{2}\right)
\end{array}\right)\left(\begin{array}{l}
M_{M}\left(a_{n}, b_{n}\right) \\
M_{N}\left(a_{n}, b_{n}\right) \\
N_{M}\left(a_{n}, b_{n}\right) \\
N_{N}\left(a_{n}, b_{n}\right)
\end{array}\right)
$$

Write $K=$

$$
\begin{gathered}
\left(\begin{array}{cccc}
M_{M}\left(a_{2}, b_{2}\right)+M_{N}\left(a_{2}, b_{2}\right) & 0 & M_{M}\left(a_{2}, b_{2}\right) & 0 \\
0 & M_{M}\left(a_{2}, b_{2}\right)+M_{N}\left(a_{2}, b_{2}\right) & 0 & M_{M}\left(a_{2}, b_{2}\right) \\
N_{M}\left(a_{2}, b_{2}\right) & 0 & N_{M}\left(a_{2}, b_{2}\right) & 0 \\
0 & N_{M}\left(a_{2}, b_{2}\right) & 0 & N_{M}\left(a_{2}, b_{2}\right)
\end{array}\right) \\
\operatorname{Then~}\left(\begin{array}{l}
M_{M}\left(a_{n}, b_{n}\right) \\
M_{N}\left(a_{n}, b_{n}\right) \\
N_{M}\left(a_{n}, b_{n}\right) \\
N_{N}\left(a_{n}, b_{n}\right)
\end{array}\right)=K^{n-2}\left(\begin{array}{l}
M_{M}\left(a_{2}, b_{2}\right) \\
M_{N}\left(a_{2}, b_{2}\right) \\
N_{M}\left(a_{2}, b_{2}\right) \\
N_{N}\left(a_{2}, b_{2}\right)
\end{array}\right)
\end{gathered}
$$

The number of discrete Morse $k$-functions on $\mathrm{P}_{\mathrm{n}}$ is the sum of all the entries in $\left(M_{M}\left(a_{n}\right)+\right.$ $\left.M_{N}\left(a_{n}\right)+N_{M}\left(a_{n}\right)+N_{N}\left(a_{n}\right)\right)$.

The number of discrete Morse $k$-functions on $C_{n}$ is $\operatorname{Tr}\left(M_{M}\left(a_{n+1}\right)+M_{N}\left(a_{n+1}\right)+N_{M}\left(a_{n+1}\right)\right)$.

This follows from the fact that we can attach the two ends of the path only if their value is equal and at least one of them is critical.

The number of discrete Morse 4-functions on $C_{n}$ for $n \in\{2, \ldots, 6\}$ are 46, 336, 2318, 16071, 111520.

\section{Further Questions}

(1) Are there formulae for the number of discrete Morse $k$-functions for other classes of graphs? (e.g. complete, complete bipartite, etc.) 
(2) Is there an efficient algorithm for computing the number of discrete Morse $k$-functions on general graphs?

(3) How many gradient vector fields and discrete Morse $k$-functions are there on $C_{n}$ up to symmetry by $Z_{n}$ ? By $D_{2 n}$ ?

(4) How many gradient vector fields and discrete Morse $k$-functions are there on higher dimensional regular $\mathrm{CW}$-complexes? On the standard $\mathrm{CW}$-complex for $M_{g}$ ?

\section{References}

Bauer, U., Lange, C. \& Wardetzky, M. (2012) Optimal topological simplification of discrete functions on surfaces. Discrete \& Compututational Geometry, 47(2), 347-377. https://doi.org/10.1007/s00454011-9350-z

Chari, M. K., \& Joswig, M. (2005). Complexes of discrete Morse functions. Discrete Mathematics, 302(1-3), 39-51. doi:10.1016/j.disc.2004.07.027

Forman, R. (1998). Morse Theory for cell complexes. Advances in Mathematics, 134(1), 90-145. doi:10.1006/aima.1997.1650

Lewiner, T., et al. (2004). Applications of Forman's Discrete Morse Theory to topology visualization and mesh compression. IEEE Transactions on Visualization and Computer Graphics, 10(5), doi:10.1109/tvcg.2004.18. 